ÁREA ABIERTA. Vol. 14. № 2. Julio 2014

http://dx.doi.org/10.5209/rev_ARAB.2014.v35.n2.45753

“LA INDUSTRIA PERIODÍSTICA EN ESTADOS UNIDOS:

AUGE Y COLAPSO DEL MODELO ECONÓMICO"

AUTOR: Dr. Manuel GOYANES MARTÍNEZ

Universidad Carlos III, Madrid. España

\title{
La industria periodística en \\ Estados Unidos: auge y colapso del modelo económico
}

The USA newspaper industry: Boom and bust of the economic model 


\section{RESUMEN:}

Los cambios tecnológicos y de comportamiento emergentes en la última década, derivados del nacimiento del periodismo digital, han provocado una profunda transformación en las organizaciones periodísticas tradicionales. Los efectos más notables han sido, sobre todo, la ruptura de la tendencia alcista de la circulación de periódicos diarios, de la inversión publicitaria impresa y del gasto diario en periódicos. En contrapartida, observamos un continuo aumento de la inversión publicitaria digital correlativamente al aumento de la audiencia de sus diarios. Pese a la importancia del estudio de los efectos de la prensa digital en la prensa impresa y del análisis de sus tendencias a largo plazo, existe una más que notable carencia de investigaciones rigurosas que asienten unas bases empíricas fiables. El presente artículo tiene como objetivo conocer la evolución, las tendencias y relaciones de diferentes variables (gasto total, circulación, inversión publicitaria, etc.) de la prensa impresa en Estados Unidos en los últimos 60 años. Asimismo, analiza los efectos de la prensa digital en la prensa impresa durante los últimos 10 años y compara la evolución de la circulación impresa de dos series temporales: por un lado la integrada dentro de los últimos 60 años y por otro, la de los últimos 10 años. Las conclusiones más destacadas extraídas del análisis empírico apuntan a que el gasto en prensa impresa afecta positivamente a su inversión publicitaria y establece una correlación significativa negativa entre el gasto en prensa impresa y la inversión publicitaria impresa y una alta correlación negativa entre la inversión publicitaria impresa y la digital.

\section{Palabras clave:}

Prensa impresa, prensa digital, inversión publicitaria, circulación de periódicos, gasto en prensa.

\section{ABSTRACT:}

The technological and behavioural changes emerging in the last decade due to the birth of digital journalism have caused a profound change in traditional news organisations. The most notable effects have been, above all, the break in the upward trend in daily newspaper circulation, investment in print advertising and daily spending on newspapers. In contrast, we observed a continuous increase in digital advertising investment in correlation to the growth of its readership. Despite the importance of studying the effects of digital media on print media and analysing its long-term trends, there is a notable lack of rigorous research establishing a reliable empirical foundation. This article aims to determine the evolution, trends and relationships of different variables (total expenditure, circulation, investment in advertising, etc.) of print media in the United States over the last 60 years. It also analyses the effects of digital media on print media over the past 10 years and compares the evolution of print circulation of series from two time periods: one built up during the last 60 years and the other, during the last 10. The main conclusions drawn from the empirical analysis point that the spending on print media positively affects its 
advertising investment, and establishes a significant negative correlation between spending on print media and investment in print advertising, and a high negative correlation between printed and digital advertising investment.

\section{Keywords:}

Traditional newspapers, digital newspapers, advertising expenditure, newspaper circulation, circulation expenditure. 


\section{Introducción}

La industria periodística ha sido tradicionalmente un sector con un margen de beneficios superior a otros sectores o industrias (Picard, Grönlund y Pönni, 2000; Picard, 2002; 2003). En la última década, sin embargo, la difusión y desarrollo del periodismo digital (López, 2010) han provocado cambios en los modos de consumo de los medios de comunicación tradicionales (Chyi y Sylvie, 2001; Campos, 2010) así como una profunda transformación de su cadena de valor en el nuevo ciberespacio (López, 2010; Goyanes, 2012, 2013b). Los efectos más notables han sido, sobre todo, la ruptura de la tendencia alcista de la circulación de periódicos diarios, de la inversión publicitaria impresa y del gasto diario en periódicos (OCDE; 2010; WAN, 2010, 2011; Pew Reseach, 2012a). En contrapartida, observamos un continuo aumento de la inversión publicitaria digital correlativamente al aumento de la audiencia de sus diarios (Curran, 2010).

Numerosas investigaciones (Sánchez-Tabernero, 2008; Curran, 2010; Larrañaga-Rubio, 2010) han profundizado en el análisis evolutivo de la prensa impresa atendiendo a distintas variables (difusión, circulación, inversión publicitaria, modelo de negocio, etc.). Los resultados apuntan a conclusiones bastante nítidas: la industria periodística sufre las consecuencias de una profunda conversión industrial provocada por la innovación disruptiva (Schumpeter, 1975; Christensen, 1997) que supone Internet y el periodismo digital (Picard, 1997, 2003; Doyle, 2002). El resultado es un acrecentado descenso continuado de múltiples indicadores económicos de la prensa impresa (Borrell, 2007; Stone, 2007), y un ascenso menormente acentuado de los indicadores económicos digitales (Kubas y Kubas, 2007). Esto provoca que las pérdidas del sector impreso no sean compensadas, de largo, por el sector digital (Pew Reseach Center, 2012a).

Pese a la importancia del estudio de los efectos de la prensa digital en la prensa impresa y del análisis de sus tendencias a largo plazo, existe una más que notable carencia de investigaciones rigurosas que asienten unas bases empíricas fiables. El presente artículo tiene como objetivo conocer la evolución, las tendencias y relaciones de diferentes variables (gasto total, circulación, inversión publicitaria, etc.) de la prensa impresa en Estados Unidos en los últimos 60 años. Asimismo, analiza los efectos de la prensa digital en la prensa impresa durante los últimos 10 años y compara la evolución de la circulación impresa de dos series temporales: por un lado, la integrada dentro de los últimos 60 años y por otro, la de los últimos 10 años.

\section{Marco teórico e hipótesis}

La crisis que ha irrumpido en nuestras vidas de modo repentino sobre el año 2008 no ha sido únicamente de naturaleza financiera o económica (Volcker, 2001). Se trata de una crisis que se podría calificar como interdependiente (Cardoso, 2011) y que se manifiesta en cuatro dimensiones principales de forma simultánea y en un intervalo de tiempo similar, aunque durante un tiempo previsto (Castells, 2000; Soros, 2001) la principal crisis se 
desarrolla en las dimensiones financiera y económica (Krugman, 2008) pero también política (Beck, 2001), medioambiental (Giddens, 1999) y comunicativa (Silverstone, 2006).

En algunos sectores, como el mediático, la crisis no es sólo coyuntural sino estructural (Larrañaga-Rubio, 2010), procedente de mucho más atrás del colapso del sistema financiero (Campos, 2010; Zallo, 2011). El sector de la comunicación se ve afectado simultáneamente por los problemas cíclicos (De Mateo y Bergés, 2011) macroeconómicos (crisis) que se cruzan con sus propias dinámicas microeconómicas de carácter estructural (Zallo, 2011).

De acuerdo a la literatura, la doble crisis que sufre el sector comunicativo se produce como consecuencia de la transformación que introducen las nuevas tecnologías (López y Pereira, 2008; Díaz-Noci, 2009) (convergencia digital, multiplicación de la competencia, mutaciones en los usos sociales, etc.) en el mismo momento en que se producen diversas circunstancias problemáticas: reducción de los ingresos publicitarios (Timoteo, 2010), dificultades en la obtención de crédito (Campos, 2010) y debilitación de los modelos de negocio tradicionales (De Mateo y Bergés, 2011; Goyanes, 2012). La afluencia de mutaciones tecnológicas, comunicativas y sociales completan la estructura fragmentada y compleja del sistema actual de información y comunicación que Roger Fidler (1997) definió como "Mediamorphosis" y Octavio Islas (2009), deudor de la ecología de medios de Marshal McLuhan, como "remediación digital" en la que nuevos y viejos medios se ven modificados por el impacto de los otros.

El debilitamiento de los modelos de negocio tradicionales (Keller, 2009) y la relativa deficiencia del modelo gratuito en Internet (Cebrián, 2010, Goyanes, 2013b) está provocando que los medios de comunicación aceleren su necesidad de buscar nuevas fuentes de ingresos (Usher, 2009) y reducir sus costos empresariales (The Economist, 2006), lo que se traduce en la exploración y ensayo de nuevos modelos de negocio destinados al pago del lector final (Goyanes, 2012) o en la combinación de estos con estrategias tradigitales (Timoteo, 2010). Las TICs son las principales aliadas con las que los grupos mediáticos operan para completar la financiación que no alcanzan los modelos de ingresos tradicionales (De Mateo y Bergés, 2011 ).

La crisis golpea duramente la prensa tradicional, que no sólo ve reducida o estancada su difusión sino también sus inversiones publicitarias (WAN, 2010; Pew Research, 2012b). Esta situación, que no sólo es europea sino generalizada en los países más desarrollados (OCDE, 2010), ha reabierto tanto los debates sobre las ayudas a la prensa como la búsqueda de nuevos modelos de propiedad (cambios en el capital de las empresas mediáticas), de financiación (fundaciones, patrocinio) y de negocio (distribución gratuita, combinada o paywall). Asimismo, ha conducido a investigadores como Philip Meyer (2009) o a consultoras como Ross Dawson (2010) a pronosticar el fin de la prensa escrita. Por lo tanto, podríamos afirmar hipotéticamente que:

H1. La media de circulación total diaria es mayor en la serie 1951/2011 que en la 2001/2011. 
La prensa tradicional basa su sistema de ingresos en el doble financiamiento (two-side market, Picard, 1999; Rochet y Tirole, 2003), que implica tanto la venta del producto al lector como el recurso de la publicidad (Sonnac, 2009). Sin lectores de prensa no hay inserciones publicitarias, puesto que es el valor percibido por las empresas para anunciarse (Bustos, 2010). Entonces:

H2. Cuanto mayor es el gasto en prensa impresa mayor es la inversión publicitaria en prensa impresa (análisis 1951/2011).

La innovación disruptiva que supone el periodismo digital para el periodismo impreso (Picard, 1999, 2003; Pavlik, 2000; Brody, 2000; Cea Estervelas, 2010), ha generado un trasvase tanto de inversiones publicitarias como de audiencia hacia lo online. Numerosos estudios han demostrado cómo el carácter gratuito de muchas publicaciones digitales ha contribuido a acentuar esta tendencia (Nesbitt y Laine, 2004; Cohen, 2007; Pfanner, 2007). De este modo, podríamos afirmar que:

H3. El gasto en prensa impresa y la inversión publicitaria en prensa digital tienen una correlación significativa negativa (análisis 2001/2011).

H4. La inversión publicitaria en prensa impresa y la inversión publicitaria digital tienen una correlación significativa negativa (análisis 2001/2011).

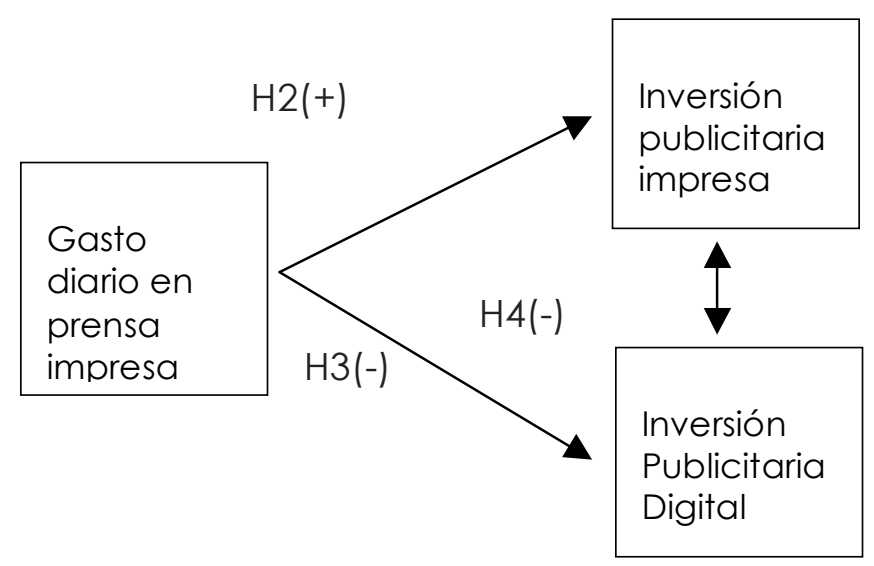

Gráfico $n^{\circ} 1$. Hipótesis de partida

\section{Objetivos y metodología}

El presente artículo tiene como objetivo conocer la evolución, las tendencias y relaciones de diferentes variables de la prensa impresa en Estados Unidos en los últimos 60 años. Asimismo, analiza los efectos de la inversión publicitaria de la prensa digital en la prensa impresa y compara la evolución de la inversión publicitaria impresa y digital en los últimos 10 años y la evolución de la circulación impresa de dos series temporales: por un lado la integrada dentro de los últimos 60 años y por otro, la de los últimos 10 años. Concretamente, la presente investigación se centra en las siguientes variables: gasto diario en prensa impresa (GDI), número total de periódicos diarios impresos (NPI), 
circulación diaria total impresa (Cl), publicidad nacional (PN), publicidad minorista (PM), publicidad clasificada (PC), inversión publicitaria impresa (IPI), inversión publicitaria digital (IPD) y total inversión publicitaria impresa y digital (IVID).

La investigación se divide en dos series temporales por la imposibilidad de recoger los datos referentes a la inversión publicitaria digital y la total impresa y digital antes del año 2001 (por razones obvias, puesto que apenas existían cibermedios y compañías medidoras de audiencias fiables). Los datos de todas las variables proceden de la Newspaper Association of America (NAA) publicados en su página web. El presente artículo, por tanto, establece dos series temporales integradas dentro del marco 1951/2011 y 2001/2011, con el objetivo de comparar resultados y establecer las correlaciones fundamentales entre las diferentes variables de cada serie temporal. En definitiva, se trata de una investigación que se define por su carácter eminentemente cuantitativo, exploratorio y longitudinal con un total de 60 observaciones para la serie 1951/2011 y 10 para la 2001/2011.

\section{Resultados}

Como observamos en la tabla uno, existen diferencias significativas respecto a las medias de las diferentes variables en las dos series temporales. Por un lado podemos observar como las medias de las variables GDI ( $M=6.607 .096), P N(M=6.562), P M(M=19.194), P C$ $(M=12.759)$ e IPI $(M=38.515)$ son mayores en la serie temporal $01 / 11$ que en la $51 / 11$. Sin embargo, la media NPI ( $M=1.653)$ y de la $\mathrm{Cl}(M=58.457)$ son mayores en la serie temporal 51/11 que en la 51/11. De estos datos se contrasta positivamente la hipótesis 1, puesto que la circulación impresa es menor en la serie 01/11 que en la 51/11, indicando una tendencia constante a la baja en los últimos 10 años.

\begin{tabular}{|c|c|c|c|c|}
\hline Variable & $\begin{array}{c}\text { M serie } \\
\mathbf{5 1 / 1 1}\end{array}$ & $\begin{array}{c}\text { M serie } \\
\mathbf{5 1 / 1 1}\end{array}$ & M serie 01/11 & DT serie 01/11 \\
\hline GDI & 4.146 .907 & $288.167,3$ & 6.607 .096 & $81.364,6$ \\
\hline $\mathrm{NPI}$ & 1.653 & 16,8 & 1.425 & 10,7 \\
\hline $\mathrm{Cl}$ & 58.457 & 554,5 & 50.612 & $1.429,5$ \\
\hline $\mathrm{PN}$ & 2.923 & 309,8 & 6.562 & 588,4 \\
\hline $\mathrm{PM}$ & 10.117 & 977,0 & 19.194 & $1.412,5$ \\
\hline $\mathrm{PC}$ & 6.788 & 805,7 & 12.759 & $1.770,3$ \\
\hline $\mathrm{IPI}$ & 19.829 & 2072,6 & 38.515 & $3.746,5$ \\
\hline $\mathrm{IPD}$ & - & - & 2.464 & 275,2 \\
\hline $\mathrm{IVID}$ & - & - & 40.979 & $3.583,9$ \\
\hline
\end{tabular}

Tabla $n^{\circ} 1$. Estadísticos descriptivos serie temporal 1951/2011 y 2001/2011.

Fuente: Elaboración propia 


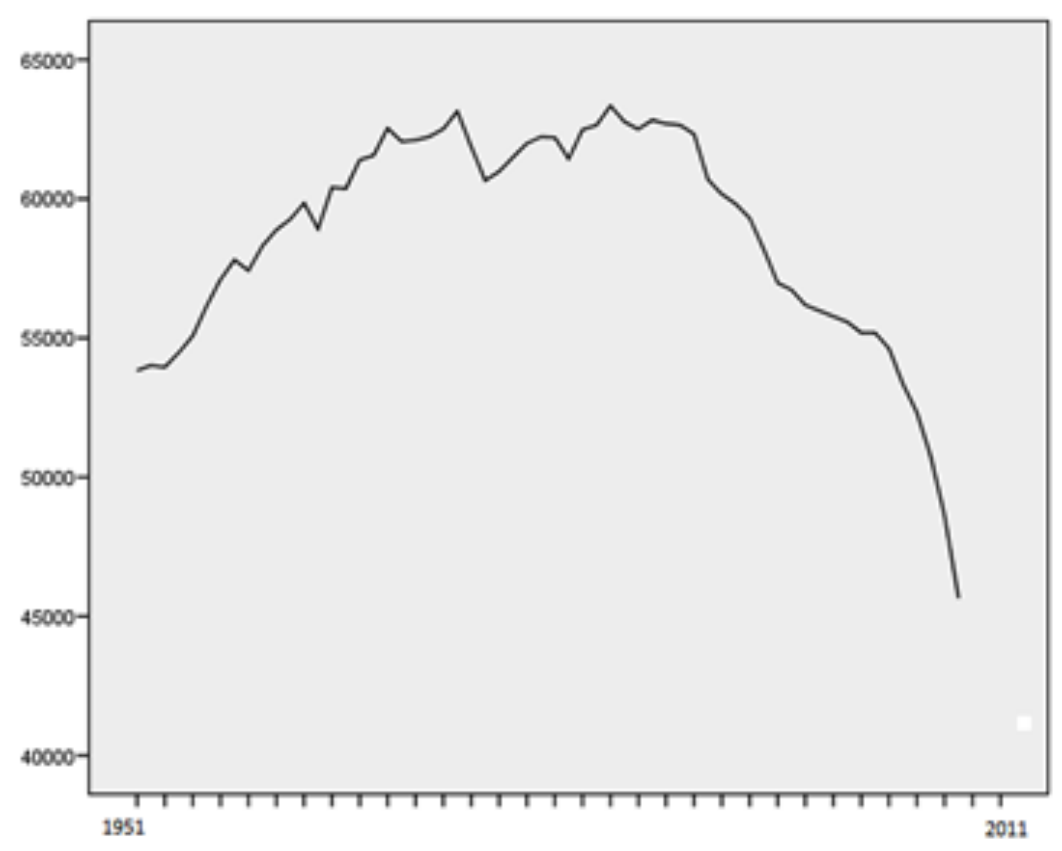

Gráfico $n^{\circ}$ 2. Circulación diaria de periódicos impresos (000) en Estados Unidos durante 1951-2011.

Fuente: Elaboración propia.

En el gráfico nº 2 podemos observar nítidamente la evolución de $\mathrm{Cl}$ (circulación impresa) en Estados Unidos en los últimos 60 años $(\mathrm{N}=60)$. La $\mathrm{Cl}$ alcanza su apogeo a finales de los años 80, década asimismo donde observamos un punto de inflexión en el que comienza su decadencia hasta la actualidad. En 2011 los datos son claros: la Cl en Estados Unidos alcanza unas cifras récord negativas, puesto que en la serie temporal analizada no hay datos en donde la circulación sea tan baja, superando incluso, los datos de 1951.

La comparación entre la $\mathrm{Cl}$ y la IPI (inversión publicitaria impresa) se muestra en el gráfico tres. En ella podemos observar cómo a lo largo de los años ( $N=60)$ la tendencia de ambas variables no es correlativa. De hecho, ambas variables tienen una correlación significativa negativa al 0,05 nivel de confianza $(r=-, 295)$ durante la serie 1951-2011 como se muestra en la tabla dos. Esto significa que cuanto más asciende la IPI a lo largo de la serie temporal menor es la Cl, o viceversa. Sin embargo, en la serie temporal 2001-2011 la correlación es significativa positiva al nivel 0,01 de confianza $(r=, 956)$, lo que significa que, en este caso, cuanto más baja la $\mathrm{Cl}$, más baja la IPI, o viceversa. Esta evolución demuestra como ambas variables experimentan, en los últimos años, una tendencia bastante similar, aunque el descenso de la IPI es bastante más acentuada.

La tendencia alcista de la IPI en Estados Unidos experimenta una inflexión a inicios del nuevo siglo, motivado por el descenso de la publicidad minorista, nacional, y, sobre todo, clasificada. La inversión publicitaria total impresa toca techo en el año 2000 con un total de 48.670 millones de dólares, mientras que ya en el 2011 se retrotrae a los 20.692 millones de dólares, es decir, en cifras aproximadamente de 1983 (20.581). La inversión total en publicidad impresa en la serie temporal 1951-2011 en Estados Unidos asciende a 1.232.738 millones de dólares mientras que en los últimos diez años (2001-2011) asciende a 140.243 millones de dólares. 


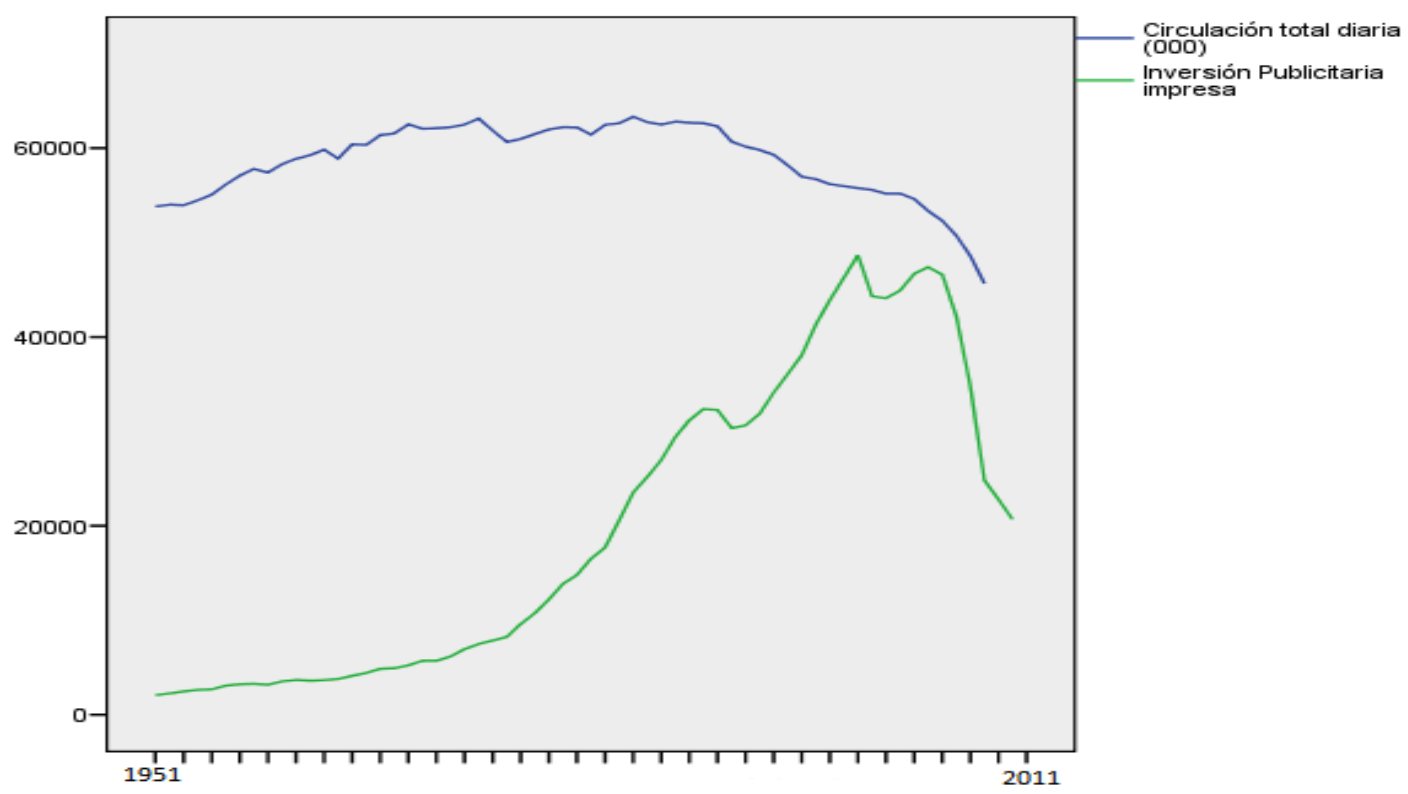

Gráfico $n^{\circ}$ 3. Evolución de la circulación diaria de periódicos impresos (000) e inversión publicitaria en prensa impresa en EE.UU. durante 1951-2011.

Fuente: Elaboración propia.

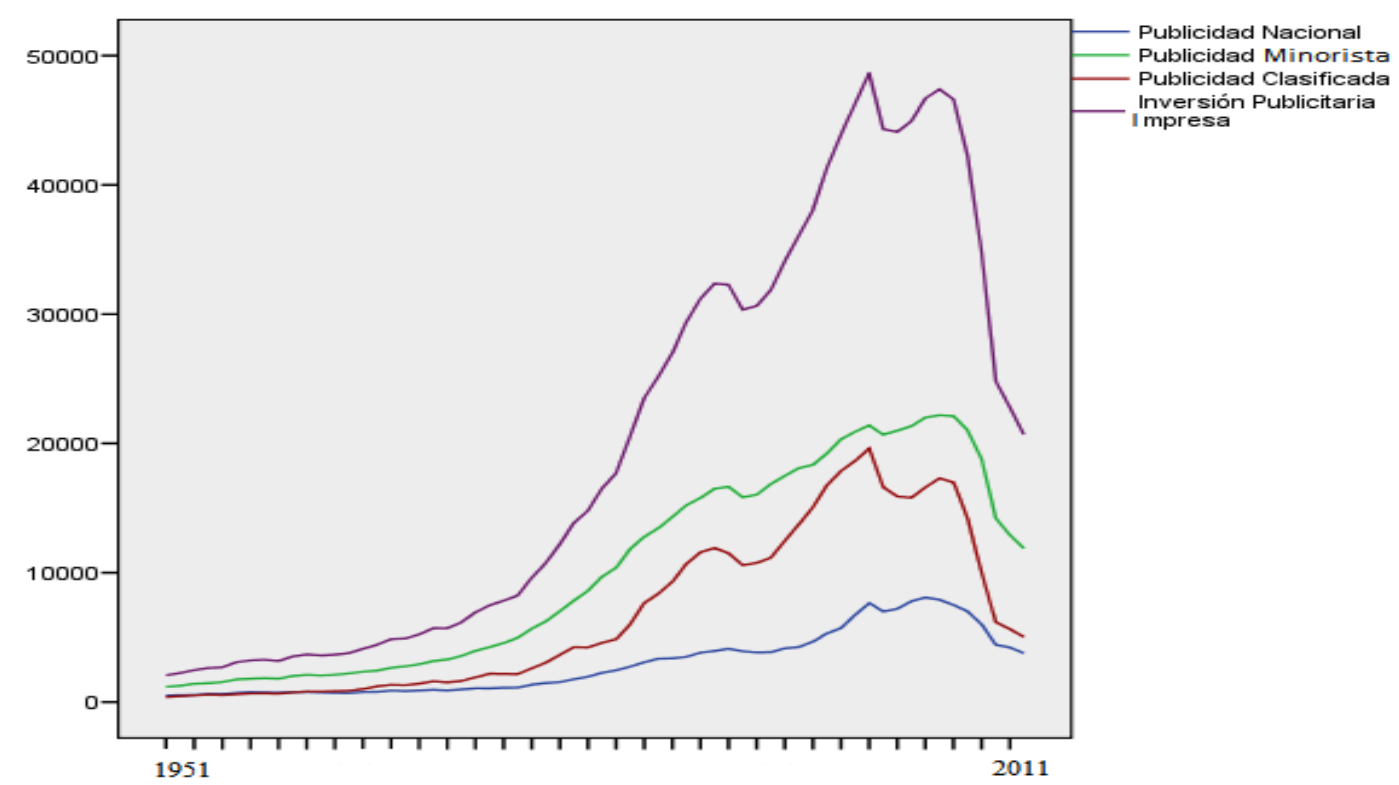

Gráfico $n^{\circ}$ 4. Evolución desde 1951 hasta 2011 de la inversión publicitaria en periódicos impresos en EE.UU. Fuente: Elaboración propia.

Al comparar (gráfico $n^{\circ} 5$ ) las inversiones publicitarias en prensa impresa y el total de inversiones en publicidad en prensa impresa y en prensa digital (es decir la suma de las dos variables), el gráfico no deja lugar a dudas: las organizaciones periodísticas actuales en Estados Unidos dependen fuertemente de las inversiones publicitarias impresas frente a las digitales. En el año 2011, de los 23.941 millones de dólares invertidos en publicidad en periódicos impresos y digitales, 20.691 corresponden a inversiones publicitarias en prensa 
impresa $(86,4 \%)$, mientras tan sólo 3.249 han correspondido a las inversiones publicitarias en prensa digital (13,6\%). Por ello, pese a que las inversiones publicitarias en prensa digital han experimentado un incremento notable, no han compensado los descensos en inversiones en prensa impresa. De hecho, como observamos en el gráfico, el intervalo existente entre las dos líneas de tendencia corresponde a las inversiones publicitarias digitales, por lo que podemos inferir claramente su insignificancia respecto a la inversión en prensa tradicional y al total de inversiones.

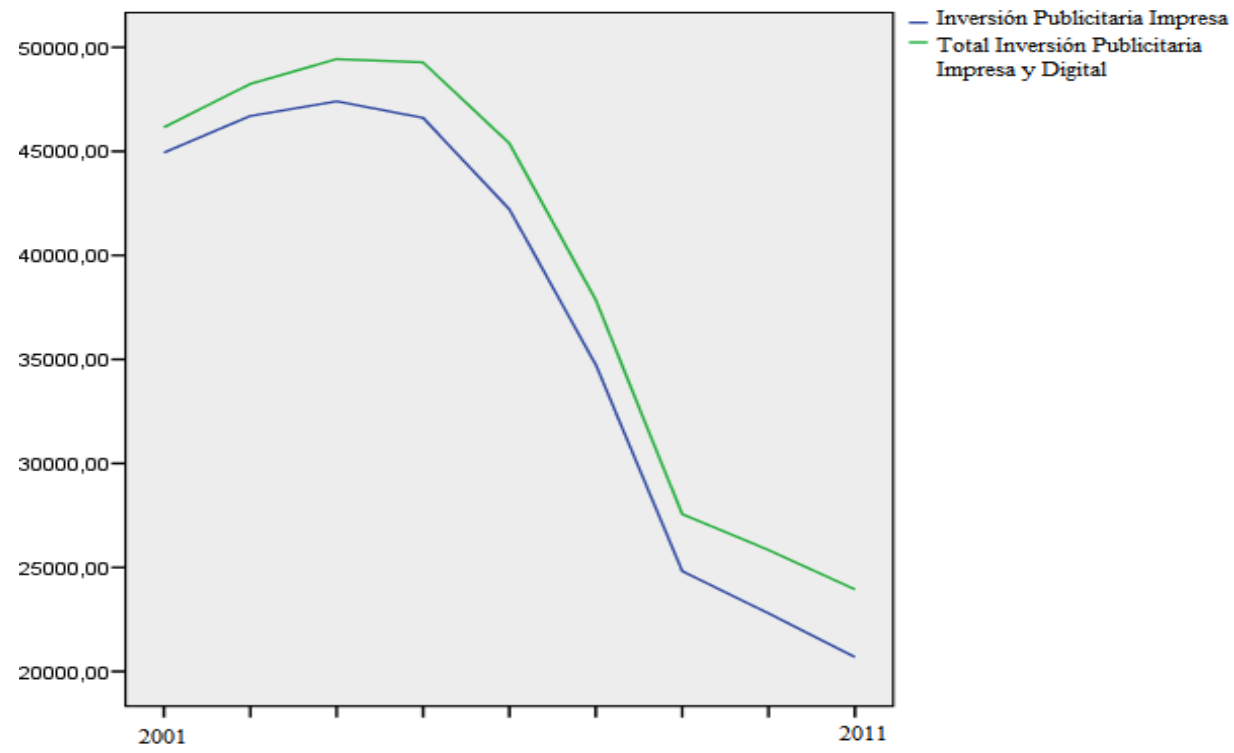

Gráfico $n^{\circ}$ 5. Evolución de las inversiones publicitarias en prensa impresa y de las inversiones publicitarias en prensa impresa y digital (suma de las dos variables).

Fuente: Elaboración propia.

Para que la explicación sea más clara y visualmente comprensible, mostramos a continuación un diagrama de cajas y bigotes en donde representamos la inversión publicitaria en prensa impresa y en prensa digital a lo largo de la serie temporal 2001/201 1 $(N=10)$. Podemos observar, en la caja de la izquierda, cómo a pesar de la tendencia negativa de la inversión publicitaria impresa, el año en donde la inversión fue más baja (dato mínimo, corresponde precisamente al 2011), con un total de 20.691 millones de dólares, se sitúa a una gran distancia respecto al dato máximo de la inversión publicitaria digital, es decir, los 3.249 millones de dólares del año 2011.

Por ello, concluimos que a pesar del aumento de los nuevos ingresos digitales, las organizaciones periodísticas en Estados Unidos dependen de los ingresos publicitarios impresos para mantener su estructura como organización (gráfico $n^{\circ} 7$ ). Esta situación podría revertirse evolutivamente a través de: 1) la implementación de nuevas estrategias de pago por contenidos en el sector digital mediante nuevos modelos de ingresos que combinen la publicidad y el pago del lector, 2) un cambio de cultura empresarial que priorice lo digital frente a lo impreso y 3) una transformación organizativa en donde el departamento de marketing (venta de subscripciones, satisfacción del cliente, necesidades del cliente, etc.) y los servicios complementarios (servicios de 
entretenimiento, cultura $u$ ocio) sean fundamentales junto al contenido producido con el objetivo de atraer y fidelizar a un cliente de pago.

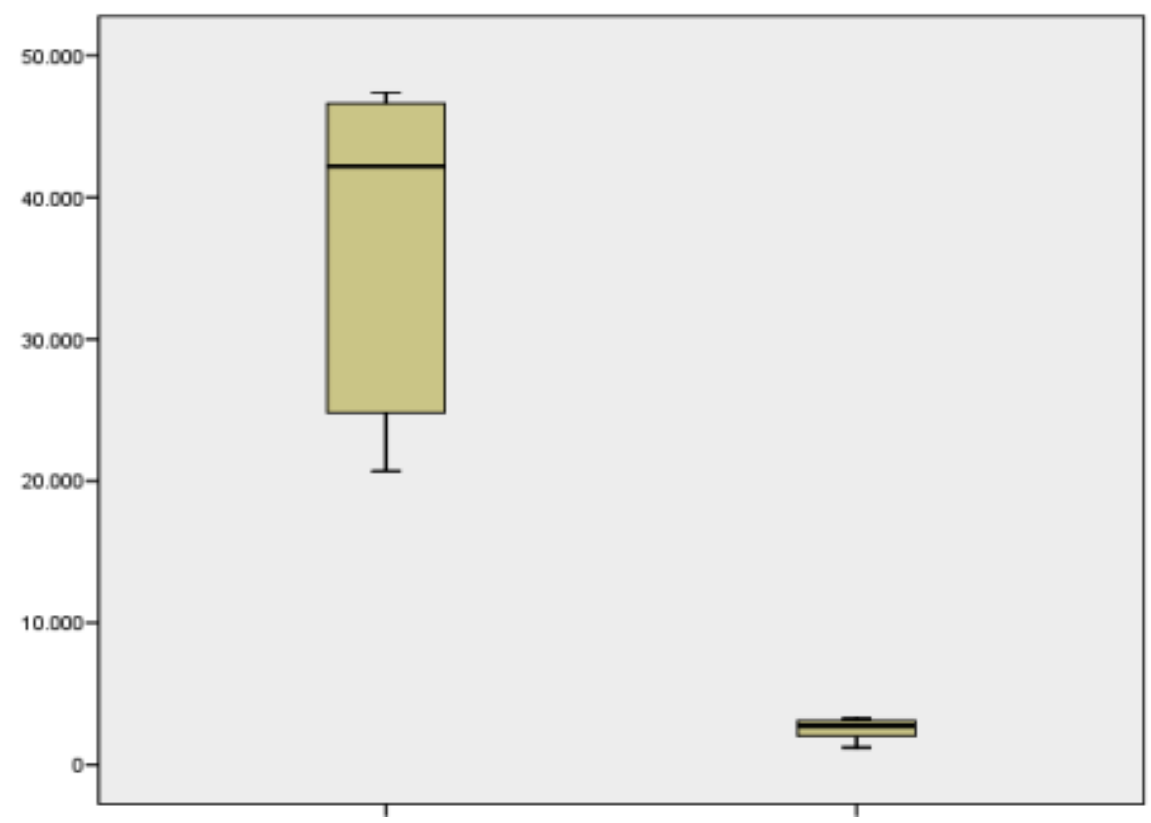

Gráfico $n^{\circ}$ 6. Diagrama de cajas de la inversión publicitaria en prensa impresa y digital. La caja de la izquierda representa la inversión publicitaria en prensa impresa y la de la derecha la digital.

Fuente: Elaboración propia.

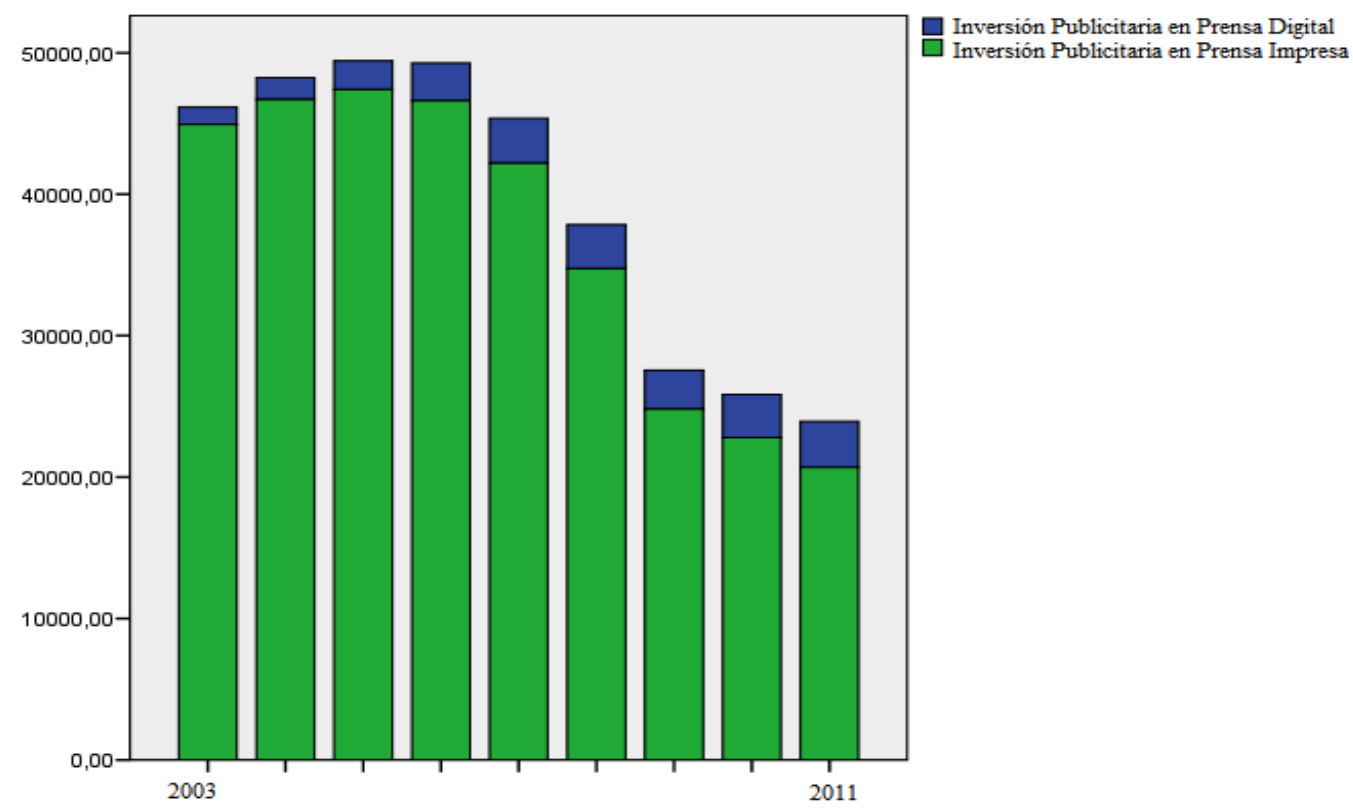

Gráfico $n^{\circ}$ 7. Evolución de la inversión publicitaria en prensa impresa y digital durante 2003/2011.

Fuente: Elaboración propia. 
Las tablas $n^{\circ} 2$ y 3 muestran las correlaciones entre las diferentes variables en las dos series temporales. Por un lado en lo que al intervalo 51/11 respecta, podemos observar cómo la correlación entre GDI e IPI es positiva y significativa $(r=, 942)$. De este modo podemos contrastar positivamente las hipótesis 2. Por otro lado, la serie temporal 01/11 muestra cómo la correlación entre GSI e IPD es negativa y significativa $(r=-, 936)$ y cómo asimismo la correlación entre IPD e IPI es negativa pero no significativa $(r=-, 645)$. De este modo podemos contrastar positivamente la hipótesis 3, pero no la 4 puesto que la correlación no es significativa ni al 0,05 ni al 0,01 de nivel de confianza. A continuación, explicamos las causas.

A pesar de que la correlación entre la IPD e IPI es negativa, ésta no es lo suficientemente significativa para que podamos aceptar empíricamente la hipótesis 4. Sin embargo, esto puede ser causa de la limitación del espacio temporal medido en las correlaciones (recordemos que se trata de los últimos 10 años) y por otro lado, a la evolución de las dos variables, dado que el descenso de una (IPI) es mayor que el aumento de la otra (IPD). De hecho, estudios de mercado (Pew Research Center, 2012a) muestran cómo a pesar de que el total de ingresos por publicidad digital aumentó un promedio del $19 \%$ el pasado año en EE.UU., no fueron suficientes para compensar las pérdidas como resultado del descenso del $9 \%$ en la publicidad de la prensa tradicional. La proporción de desplazamiento fue una pérdida de aproximadamente de 7 a 1 (de cada siete dólares que se perdió en la prensa impresa se ganó uno en el terreno digital). Por lo tanto, la situación es incluso peor de lo que la hipótesis planteaba.

Tabla 2. Correlaciones prensa impresa. Serie temporal 1951-2011

\begin{tabular}{|c|c|c|c|c|c|c|c|}
\hline & GDI & NPI & $\mathrm{Cl}$ & PN & PM & PC & IPI \\
\hline GDI & 1 &,$- 893\left({ }^{* *}\right)$ &,$- 489\left(^{* *}\right)$ & $.914\left({ }^{* *}\right)$ & $.968\left({ }^{* *}\right)$ & $.894\left(^{* *}\right)$ & $.942(* *)$ \\
\hline NPI &,$- 893(* *)$ & 1 & $.617\left(\left(^{* *}\right)\right.$ &,$- 927(* *)$ &,$- 901(* *)$ &,$- 862(* *)$ &,$- 898\left(^{* * *}\right)$ \\
\hline $\mathrm{Cl}$ &,$- 489\left(^{* *}\right)$ & $.617\left({ }^{* *}\right)$ & 1 & $-423(* *)$ &,$- 281\left(^{*}\right)$ &,$- 255\left(^{*}\right)$ &,$- 295\left(^{*}\right)$ \\
\hline PN & $.914\left({ }^{* *}\right)$ &,$- 927\left({ }^{* *}\right)$ &,$- 423(* *)$ & 1 & $.963(* *)$ & $.952\left({ }^{* *}\right)$ & $.974(* *)$ \\
\hline PM & $.968\left(^{* *}\right)$ &,$- 901(* *)$ &,$- 281\left(^{*}\right)$ & $.963\left({ }^{* *}\right)$ & 1 & $.973(* *)$ & $.994\left(^{* *}\right)$ \\
\hline PC & $.894(* *)$ &,$- 862\left(^{* *}\right)$ &,$\left.- 255 l^{*}\right)$ & $.952(* *)$ & $.973\left({ }^{* *}\right)$ & 1 & $.990\left(^{* *}\right)$ \\
\hline IPI & $.942\left(^{* *}\right)$ &,$- 898\left(^{(* *)}\right.$ &,$- 295\left(^{*}\right)$ & $.974(* *)$ & $.994(* *)$ & $.990\left(^{* *}\right)$ & 1 \\
\hline
\end{tabular}

${ }^{*} p<.05 ;{ }^{* *} p<.01$. Gasto diario en prensa impresa (GDI), número total de periódicos diarios impresos (NPI), circulación diaria total impresa (CI), publicidad nacional (PN), publicidad minorista (PM), publicidad clasificada (PC), inversión publicitaria impresa (IPI). Fuente: Elaboración propia 


\begin{tabular}{|c|c|c|c|c|c|c|c|c|c|}
\hline & GDI & NPI & $\mathrm{Cl}$ & PN & PM & $P C$ & IPI & IPD & IVID \\
\hline GDI & 1 & $940(* *)$ & $941\left(^{* *}\right)$ & $.869\left(^{* *}\right)$ & $.787\left(\left(^{*}\right)\right.$ & $849\left(^{* *}\right)$ & $.833\left({ }^{* *}\right)$ &,$- 936\left({ }^{* *}\right)$ & $.804\left(^{* *}\right)$ \\
\hline $\mathrm{NPI}$ & $940\left(^{* *}\right)$ & 1 & $.993(* *)$ & $.973\left({ }^{* *}\right)$ & $.916\left({ }^{* *}\right)$ & $956\left({ }^{* *}\right)$ & $.950\left(^{* *}\right)$ & $-816\left(^{*}\right)$ & $.930\left(^{* *}\right)$ \\
\hline $\mathrm{Cl}$ & $941\left(^{* *}\right)$ & $.993\left({ }^{* *}\right)$ & 1 & $.979\left(^{* *}\right)$ & $.932\left(^{* *}\right)$ & $.955\left(^{* *}\right)$ & $.956\left(^{* *}\right)$ & $-804\left(^{*}\right)$ & $.938\left(^{* *}\right)$ \\
\hline $\mathrm{PN}$ & $.869\left(^{* *}\right)$ & $.973\left({ }^{* *}\right)$ & $.979\left({ }^{* *}\right)$ & 1 & $.986\left(^{* *}\right)$ & $.989\left(^{* *}\right)$ & $.995\left(^{* *}\right)$ &,$- 704\left(^{*}\right)$ & $.989\left(^{* *}\right)$ \\
\hline PM & $.787\left(^{*}\right)$ & $.916\left({ }^{* *}\right)$ & $932(* *)$ & $.986\left({ }^{* *}\right)$ & 1 & $.979\left({ }^{* *}\right)$ & $.993(* *)$ &,- 595 & $.995\left(^{* *}\right)$ \\
\hline $\mathrm{PC}$ & $.849\left({ }^{* *}\right)$ & $.956\left({ }^{* *}\right)$ & $.955\left(^{* *}\right)$ & $.989\left({ }^{* *}\right)$ & $.979\left({ }^{* *}\right)$ & 1 & $.996\left(^{* *}\right)$ & -.659 & $.993\left({ }^{* *}\right)$ \\
\hline IPI & $.833\left(^{* *}\right)$ & $.950\left(^{* *}\right)$ & $.956\left(^{* *}\right)$ & $.995\left(^{* *}\right)$ & $.993\left(^{* *}\right)$ & $.996\left({ }^{* *}\right)$ & 1 &,- 645 & $.999\left(^{* *}\right)$ \\
\hline IPD &,$- 936\left(^{* *}\right)$ &,$- 816\left(^{*}\right)$ &,$- 804\left(^{*}\right)$ &,$- 704\left(^{*}\right)$ &,- 595 &,- 659 &,- 645 & 1 &,- 603 \\
\hline IVID & $.804\left(^{* *}\right)$ & $.930\left(^{* *}\right)$ & $.938\left({ }^{* *}\right)$ & $.989\left(^{* *}\right)$ & $.995\left(^{* *}\right)$ & $.993\left({ }^{* *}\right)$ & $.999(* *)$ &,- 603 & 1 \\
\hline
\end{tabular}

Tabla $n^{\circ}$ 3. Correlaciones prensa e impresa y prensa digital. Serie temporal 2001-2011Fuente: Elaboración propia. ${ }^{*} \mathrm{p}<.05 ;{ }^{* *} \mathrm{p}<.01$. Inversión publicitaria digital (IPD) y total inversión publicitaria impresa y digital (IVID)

\section{Conclusiones}

Pese a que la prensa impresa ha sido tradicionalmente un sector con márgenes de beneficios superiores a otras industrias o servicios (Picard, 2003), en la última década observamos una tendencia bajista alarmante tanto en la circulación de periódicos como en ingresos publicitarios (la primera variable impacta en la segunda). Esta decadencia se debe, fundamentalmente, al nacimiento de nuevos medios digitales como nuevo sistema informativo, substitutivo y complementario del mercado impreso. Estos nuevos tipos de proveedores de contenidos representan nuevas tecnologías disruptivas que emergen y desestabilizan el mercado tradicional. Los efectos en la industria periodísticas son un irremediable proceso de canibalización donde el nuevo producto, es decir, la prensa digital, adquiere parte de la demanda impresa.

La canibalización es fruto asimismo de una estrategia de monetización del contenido digital equivocada: en la mayor parte de organizaciones periodísticas el modelo de ingresos fundamental pivota en la publicidad y no en el pago del lector. De este modo, se desestima la fuente principal de ingresos en el terreno impreso en la mayor parte de organizaciones. Dado que el valor de un periódico digital en base a un sistema de ingresos fundamentado en publicidad reside en la audiencia total (medida en número de visitas únicas mensuales), el contenido pasa a ser una commodity ya que el usuario no paga, únicamente consume información (gratuitamente). Por lo tanto su satisfacción, demandas y necesidades están supeditadas a la rentabilidad cortoplacista publicitaria, aunque ésta última sea ampliamente capturada por importantes agentes digitales ajenos a la industria periodística como son Google, Yahoo o Amazon. En este sentido, sería una contribución más que apropiada nuevas investigaciones que se centren en el "valor" y su creación en organizaciones periodísticas gratuitas y con estrategias de pago por contenidos.

La presente investigación demuestra cómo el gasto en prensa impresa afecta positivamente a su inversión publicitaria $(r=942)$ y establece una correlación significativa negativa entre el gasto en prensa impresa y la inversión publicitaria impresa $(r=-, 936)$ y una alta correlación negativa $(r=-645)$ entre la inversión publicitaria impresa y la digital. Por lo tanto, a pesar de la fuerte inversión publicitaria en periódicos digitales, su aumento no es tan pronunciado como el descenso de la inversión publicitaria impresa. Y esperamos que continúe. 


\section{Limitaciones}

Una de las limitaciones del presente estudio es la propia técnica estadística empleada. Las correlaciones, al no implicar causalidad, no nos permiten conocer qué variable es causa y cuál es efecto. Esto queda reflejado en el modelo al señalar la direccionalidad con una flecha con una doble punta entre las variables, excepto en la hipótesis tres donde numerosos estudios y análisis de mercado demuestran la direccionalidad entre GPI e IPI. Por otro lado, cada una de las variables puede verse afectada por una variable tercera no medida en el presente estudio. 


\section{BIBLIOGRAFÍA}

BORRELL ASSOCIATES (2007): Best practices report: How leading newspaper sites manage sales. Portsmouth, Borrell Associates.

BRODY, Jeffrey (2000): "The Structure of the Internet Industry", en GRECO, Albert (Ed.): The media and entertainment industries: Readings in mass communications. Boston, Allyn and Bacon.

BUSTOS, Juan Carlos y CASADO DEL RíO, Miguel (2010): "Sobre pago y gratuidad. Momentos de crisis en la industria periodística". Telos, $n^{\circ} 83$.

CAMPOS, Francisco (2010): "Los nuevos modelos de gestión de las empresas mediáticas". Estudios sobre el Mensaje Periodístico, vol. 16, pp. 13-30.

CARDOSO, Gustavo (2011): "Más allá de Internet y de los medios de comunicación de masas". Telos, n57.

CASTELLS, Manuel (2000): La sociedad red. Madrid, Alianza Editorial.

CEA ESTERUELAS, Nereida (2010): "Mediación tecnológica y estructura de negocio de la información en Internet", en CABRERA, Ángeles (Ed.): Evolución tecnológica y cibermedios. Sevilla, Comunicación Social.

CEBRIÁN, Juan Luis (2010): "Conferencia Foro Innovatec" http://www.foroinnovatec.com [Fecha de consulta: 05/09/2012].

CHRISTENSEN, Clayton (1997): The innovators dilemma: When new technologies cause great firms to fail. Boston, Harvard Business School Press.

CHYI, Iris y SYLVIE, George (2001): "The medium is global, the content is not: The role of geography in online newspaper markets". Journal of Media Economics, vol. 14, n4, pp. 231-248.

CHYI, Iris; SYLVIE, George (2009): "Online newspapers in the U.S.: Perceptions of markets, products, revenue and competition". International Journal on Media Management, vol. 2, $n^{\circ} 2$, pp. 69-77.

COHEN, Heidi (2007): "What 'time on site' means for online media", en Clickz.com [Fecha de consulta: 28/08/2012].

CURRAN, James (2010): "The future of journalism". Journalism Studies, vol. 11, n 4, pp. 464476.

DE MATEO, Rosario y BERGÉS, Laura (2011): "Reflexiones sobre el proceso de cambios en la estructura de la industria de la comunicación y en la gestión empresarial" en CAMPOS, Francisco (Ed.): El nuevo escenario mediático. Zamora-Sevilla, Comunicación Social.

DÍAZ-NOCl, Javier (2009): 2018: "¿Diarios en dispositivos móviles? Libro electrónico, tinta electrónica y convergencia de la prensa impresa y digital". El profesional de la información, vol. 18, n³, pp. 301-307. 
FIDLER, Roger (1997): Mediamorphosis. Understanding new media. California, Pineforge Press.

GOYANES, Manuel (2012): "Estrategias de pago por contenidos de la prensa digital: Una aproximación teórica". Index Comunicación, vol. 2, n¹, pp. 91-112.

GOYANES, Manuel (2013a): "Estrategias y modelos de negocio: Aclaración de conceptos y terminología de la prensa en Internet". Estudios sobre el Mensaje Periodístico, vol. 19, n 1 , pp. 419-431.

GOYANES, Manuel (2013b): "Conductores de valor de la prensa digital con estrategias de pago por contenidos: análisis de caso del Financial Times, The Times y El Mundo en Orbyt". Palabra Clave, vol. 16, n 13, pp. 873-910.

ISLAS, Octavio (2009): "Internet y la obligada remediación de la televisión". Razón y Palabra, $n^{\circ} 59$.

KELLER, Bill (2010): "Talk to the newsroom: Executive Editor", en The New York Times. http://www.nytimes.com [Fecha de consulta: 4/08/2012].

KRUGMAN, Paul (2008): The return of depression economics and the crisis of 2008. New York, W.W. Norton.

LARRAÑAGA-RUBIO, Julio (2010): "Industria de los periódicos: nuevos modelos económicos y nuevos soportes". Estudios sobre el mensaje periodístico, n 16, pp. 59-78.

LÓPEZ, Xosé (2010): La metamorfosis del periodismo. Historia de lo que permance y de lo que cambia en el ciberperiodismo del tercer milenio. Zamora-Sevilla, Comunicación Social.

LÓPEZ, Xosé y PEREIRA, Xosé (2008): "La prensa ante el reto online. Entre las limitaciones del modelo tradicional y las incógnitas de su estrategia digital", en BUSTAMANTE, Enrique et al. (Eds): Alternativas en los medios de comunicación digitales. Barcelona, Gedisa.

MEYER, Philip (2009): "El periódico élite del futuro", en ESPADA, Arcadi; HERNÁNDEZ-BUSTO, Ernesto (eds.): El fin de los periódicos. Barcelona, Duomo.

NESBITT, Mary y LAVINE, John (2004): Reaching new readers: Revolution, not evolution. Evanston, Northwestern University Readership Institute.

OCDE (2010): "The evolution of news and the Internet" http://www.oecd.org/internet/ieconomy/45559596.pdf [Fecha de consulta: 25/09/2012].

PAVLIK, John (2000): "The Structure of the New Media Industry", en GRECO, Albert (Ed.): The media and entertainment industries: readings in mass communications. Boston, Allyn and Bacon.

PEW RESEARCH CENTER (2012a): "How newspapers are faring trying to build digital revenue", 
http://www.journalism.org/analysis_report/search_new_business_model [Fecha de consulta: 25/09/2012].

PEW RESEARCH CENTER (2012b): State of the news media 2012. Washington, Project for Excellence in Journalism.

PFANNER, Eric (2007): "Norwegian newspaper publisher finds the secret to profiting online". http://www.nytimes.com/2007/02/18/business/worldbusiness/18ihtpapers.4633531.html ?pagewanted=all\&_r=0 [Fecha de consulta: 25/10/2012].

PICARD, Robert y BRODY, Jeffrey (1997): The newspaper publishing industry. Boston, WANIFRA.

PICARD, Robert, GRÖNLUND, Mikko y PÖNNI, Veijo (2000): Competitiveness of the European Union publishing industries. Luxembourg, Office of Official Publications of the European Communities.

PICARD, Robert (1999): "Implications of the changing business model of the newspaper industry". International Newspaper Financial Executives Quarterly Journal, vol. 5, n 3, pp. 25.

PICARD, Robert (2002):The economics and financing of media companies. New York, Fordham University Press.

PICARD, Robert (2003). "Cash cows or entrecote: Publishing companies and disruptive Technologies". Trends in Communication, vol. 11, n², pp. 127-136.

ROCHET, Jean-Charles y TIROLE, Jean (2004): "Two-side markets: An overview". http://faculty.haas.berkeley.edu/hermalin/rochet_tirole.pdf. [Fehca de consulta: 25/02/2012].

ROSS DOWSON (2010): "Launch of Newspaper Extinction Timeline for every country in the world". http://rossdawsonblog.com/weblog/archives/2010/10/launch_of_newsp.html [Fecha de consulta: 19/02/2013].

SÁNCHEZ-TABERNERO, Alfonso (2008): "La prensa en Europa: claves de un sector estancado". Telos, n 75, pp. 104-106.

SCHUMPETER, Joseph (1975). Capitalism, socialism and democracy. New York, Harper and Row.

SILVERSTONE, Roger (2006): Media and morality: on the rise of mediapolis. Oxford, Polity.

SONNAC, Nathalie (2009): "L'economie de la presse: vers un nouveau modèle d'affaires. http://florlecam.com/slj//wpcontent/uploads/2009/01/eco_journalisme_texte_sonnac.pdf [Fecha de consulta: 18/5/2012].

SOROS, George (2001): "The new global finance architecture", en HUTTON, Will y GIDDENS, Anthony (Eds.): On the edge: Living with global capitalism. London, Vintage. 
THE ECONOMIST (2006): "Who killed the newspaper", en The Economists http://www.economist.com [Fecha de consulta: 30/09/2012].

TIMOTEO, Jesús (2010): "Propuesta para un nuevo modelo de negocio en la industria de la comunicación", en CAMPOS, Francisco (Coord.): El nuevo escenario mediático. ZamoraSevilla, Comunicación Social.

USHER, Nikki (2009): "The business model for news is and always has been broken and Rupert Murdoch can't fix it", en The Online Journalism Review.

http://www.ojr.org [Fecha de consulta: 25/06/2012].

VOLCKER, Paul (2001): "The sea of global finance", en HUTTON, Will y GIDDENS, Anthony (Eds.): On the edge: Living with global capitalism. London: Vintage.

WAN (2010): World Press Trends. París

WAN (2011): World Press Trends. París.

ZALLO, Ramón (2010): "Políticas de comunicación audiovisual en (y para después de) la crisis económica en España", en CAMPOS, Francisco (Coord.): El nuevo escenario mediático. Zamora-Sevilla, Comunicación Social. 